\title{
Epigenetic 'tags' linked to homosexuality in men
}

Twin study reveals five DNA markers that are associated with sexual orientation.

Sara Reardon

08 October 2015 I Updated: 12 October 2015

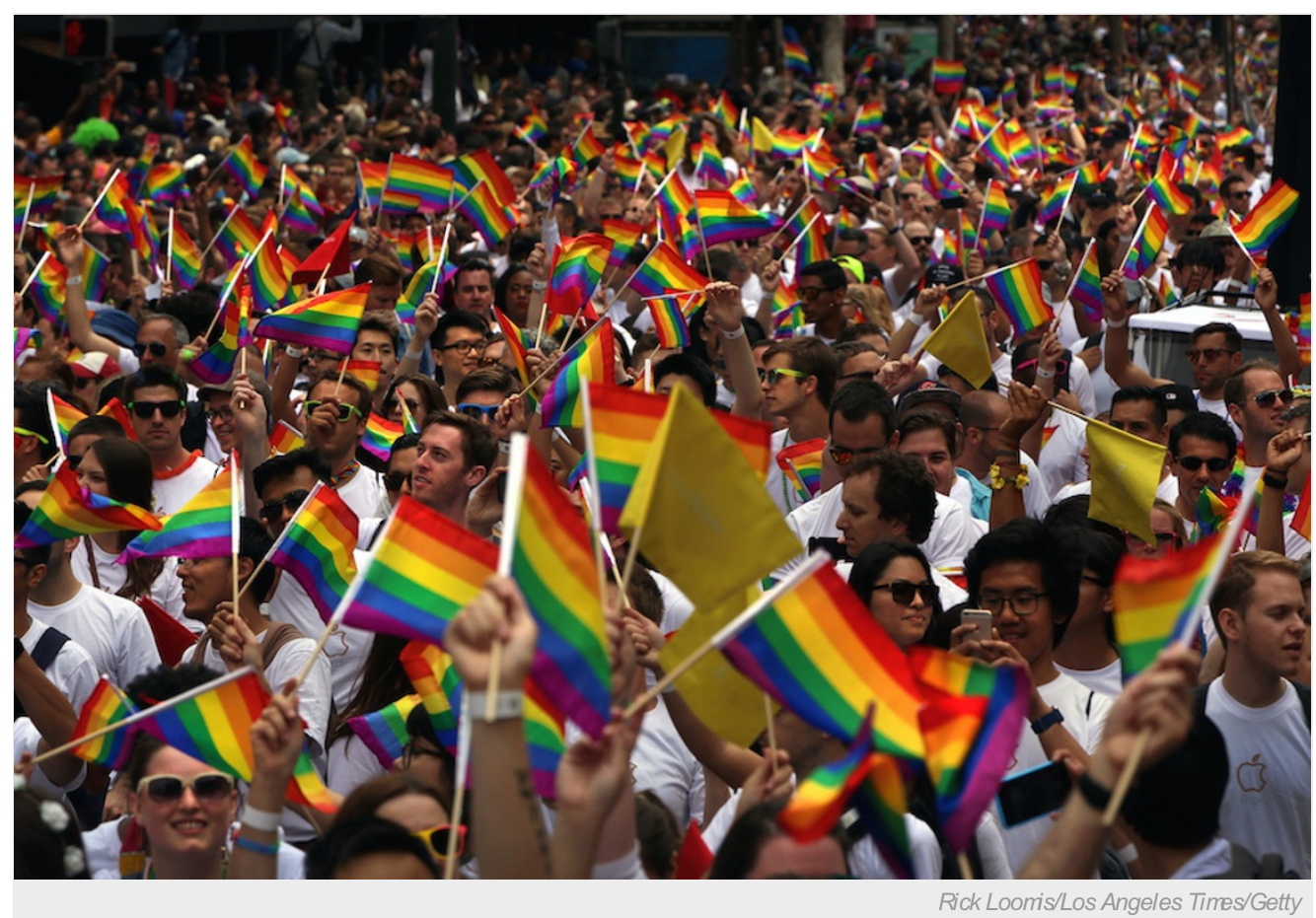

The biological influences on sexual orientation may extend to DNA 'tags' that affect gene expression.

Update, 12 October 2015: Since this story was first published, several researchers have criticized the study's methods. Some statisticians, including Andrew Gelman, at Columbia University in New York, have said that the study incorrectly presented its results as statistically significant. Study co-author Tuck Ngun, of the University of California, Los Angeles, has disputed this and other statistical criticisms, although he has acknowledged another criticism that his study was underpowered. He has not yet made the full details of his analysis available, but has said he and his collaborators will issue a statement.

The biology of sexual orientation has been one of the most vexing — and politically charged — questions in human genetics. For the first time, researchers have found associations between homosexuality and markers attached to DNA that can be influenced by environmental factors.

Twin studies and family trees provide strong evidence that sexual orientation is at least partly genetic. When one identical twin is gay, there is about a $20 \%$ chance that the other will be as well ${ }^{1}$. But because this rate is not $100 \%$, it is thought that environmental factors play a role as well. One of the best characterized is the 'older brother effect': the chance of a man being gay increases by $33 \%$ for each older brother he has ${ }^{2}$. The reason is not clear, although one hypothesis holds that the mother's immune system begins to react against male antigens and alter the fetus's development.

To search for factors that could mediate a link between environment and genes, geneticist Eric Vilain at the University of California, Los Angeles (UCLA), and his colleagues looked at epigenetic markers - chemical changes to DNA that affect how genes are expressed, but not the information they contain. These 'epi-marks' can be inherited, but can also be altered by environmental factors such as smoking, and are not always shared by identical twins.

The researchers collected DNA samples in saliva from 37 pairs of identical twins in which only one twin was gay, and 10 pairs in which both were gay. By scanning the twins' epigenomes, the researchers found five epi-marks that were more common among the gay men than in their genetically identical straight brothers. An algorithm they developed based on the five epi-marks could correctly predict the sexual orientation of men in the study $67 \%$ of the time. UCLA computational geneticist Tuck Ngun will present the work on 8 October at 
the American Society of Human Genetics meeting in Baltimore, Maryland.

Vilain is not surprised to find that epigenetics is associated with sexual orientation, although he says it is too early to try to directly link the epi-marks to any particular environmental exposure or the expression of a specific gene. Ngun says that the researchers want to replicate the study in a different group of twins and also determine whether the same marks are more common in gay men than in straight men in a large and diverse population. Associations found in small studies are prone to evaporate when tested in larger groups.

\section{Biological unknowns}

Vilain acknowledges limitations of the study — for instance, epigenetic markers differ between tissues in the body, and those in the brain are probably most relevant to sexual orientation.

But researchers welcome the addition to the sparse data on the biology of sexual orientation. "I think it certainly has the potential to add some knowledge," says psychiatric geneticist Alan Sanders at the NorthShore Research Institute in Evanston, Illinois.

Researchers also caution that just as known genetic associations offer scant insight into the mechanisms of sexual orientation, little can be concluded from the locations of the epi-marks. One mark was associated with a gene in the immune system, a second with brain development. The other three were in regions of DNA that are not associated with any gene.

"We already know there is no 'gay gene'," says William Rice, an evolutionary geneticist at the University of California, Santa Barbara. If there were, he says, it would have turned up in one of the massive studies that scan the whole genome for variants shared between gay people. The largest such study, led by Sanders, looked at 409 pairs of gay brothers including some non-identical twins. The researchers found that gay men shared similarities in two areas of the genome: the $\mathrm{X}$ chromosome and chromosome $8^{3}$. But Sanders says that he and his colleagues are still trying to determine which particular genes or other components in these regions contribute to sexual orientation.

Similarly, researchers will want to determine the biological significance of these epigenetic associations,

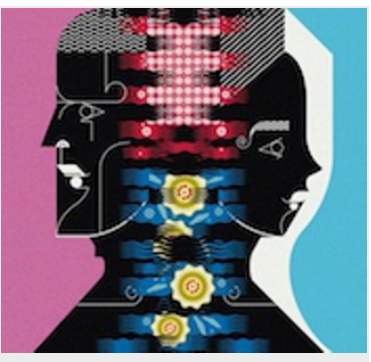

Read next: Sex redefined Rice says. Many people want to understand more about the roots of their sexual orientation, he says. "When the biology is developed, they'll at least be able to understand."

Nature | doi:10.1038/nature.2015.18530

\section{Updates}

Updated:Details discovered after the story was published have been added at the top.

\section{References}

1. Ngun, T. C., Ghahramani, N., Sánchez, F. J., Bocklandt, S. \& Vilain, E. Front. Neuroendocrinol. 32, 227-246 (2011).

2. Blanchard, R. Horm. Behav. 40, 105-114 (2001).

3. Sanders, A. R. et al. Psychol. Med. 45, 1379-1388 (2015). 